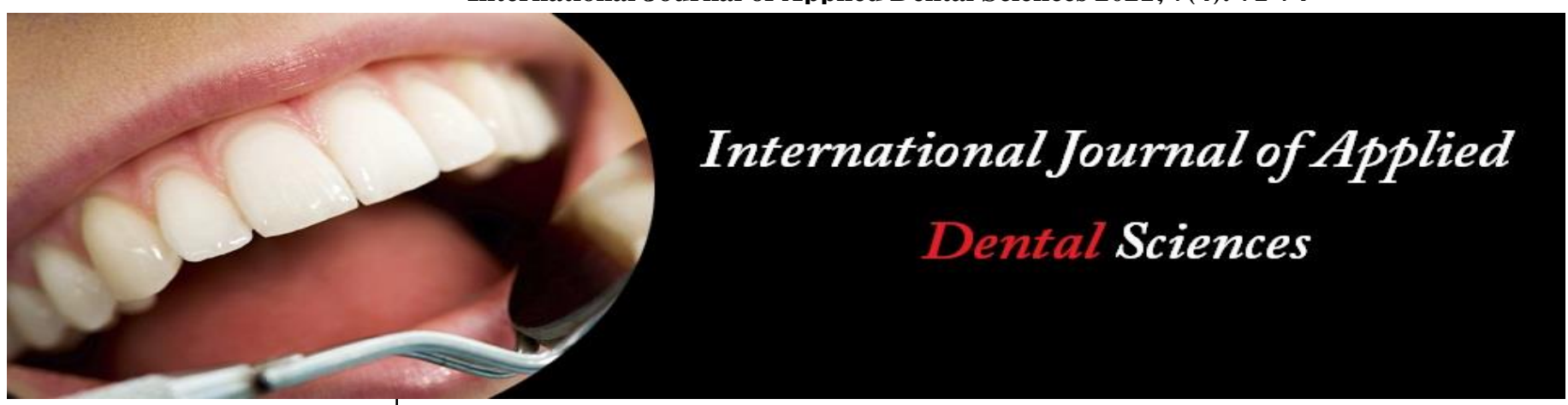

ISSN Print: 2394-7489

ISSN Online: 2394-7497

IJADS 2021; 7(4): 71-74

(C) 2021 IJADS

www.oraljournal.com

Received: 01-08-2021

Accepted: 03-09-2021

\section{Devendra Kuma}

Postgraduates Students,

Department of Prosthodontics,

Govt. Dental College,

Trivandrum, Kerala, India

\section{R Ravichandran}

Professor, Department of

Prosthodontics, Govt. Dental

College, Trivandrum, Kerala,

India

\section{Harsha Kumar K}

Professor and Head, Departmen of Prosthodontics, Govt. Dental College, Trivandrum, Kerala, India

\section{Vivek V Nair}

Professor, Department of Prosthodontics, Govt. Dental College, Trivandrum, Kerala, India
Corresponding Author: Devendra Kumar Postgraduates Students, Department of Prosthodontics, Govt. Dental College,

Trivandrum, Kerala, India

\section{Management of recurrent fracture of single removable complete denture using stainless steel mesh reinforced denture base opposing a removable partial denture: A} case report

\author{
Devendra Kumar, R Ravichandran, Harsha Kumar K and Vivek V Nair
}

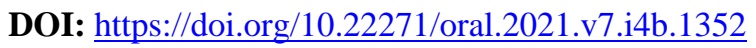

\section{Abstract}

Midline fracture is the most common fracture seen in the patient with conventional acrylic resin maxillary denture against the opposing complete or partial natural dentition. This happens due to excessive masticatory forces from the natural dentition against the opposing arch. To overcome this problem, stainless steel can be added either in the form of mesh, bar or plate.

In this present case report described the prosthetic rehabilitation of a complete maxillary edentulous and partial mandibular edentulous patient with stainless steel mesh reinforced maxillary removable complete denture and conventional removable partial denture to improve the impact strength of the denture and also at the same time to reduce the chances of alveolar ridge resorption.

Keywords: stainless steel mesh, complete denture, PMMA resin, midline fractures

\section{Introduction}

Midline fracture of the conventional maxillary denture is the most common fracture seen in the patient with opposing arch natural dentition present. Mostly the complete denture is made with the material acrylic resin; polymethyl methacrylate (PMMA). Advantages of acrylic resin are that it is easy to use, easy to repair and less time consuming. Disadvantages are low impact strength and low fatigue resistance. During chewing or performing any activities in the mouth, there is an increased masticatory force due to the opposing natural teeth. Darbar et al. have shown that the most common type of fracture is debonding/fracture of denture teeth $(33 \%)$ in both complete and partial dentures followed by the midline fractures of complete dentures $(29 \%)$ and other types $(38 \%)$ of denture fracture ${ }^{[4]}$.

Stainless steel mesh incorporated denture has a good strength which helps to prevent midline fracture of the denture. The reduced thickness of the metal mesh decreases the thickness of the acrylic denture base and improves the phonetics. Polycarbonates and polyamides although improve the strength are expensive and technique sensitive. Modification of the chemical structure by the addition of cross-linking agents like polyethylene glycol or by copolymerization with rubber in the form of Butadiene styrene has been tried. But this did not show significant enhancement on the strength properties. This case report described the prosthetic rehabilitation of a complete maxillary edentulous and partial mandibular edentulous patient with stainless steel mesh reinforced maxillary removable complete denture and conventional removable partial denture which increase fracture resistance against the forces exerted by the opposing natural dentition.

Indications of metal denture base

- Patients with increased residual ridge resorption like in conditions of diabetes mellitus, post-menopausal women.

- Patient with atrophied ridges

- Patients with compromised neuromuscular coordination, who may drop their dentures 


\section{Advantages of metal denture base}

- They have excellent strength to volume ratios and may be cast in thin sheets maintaining rigidity and fracture resistance ${ }^{[5]}$.

- Thinner metallic denture base decrease interference with phonation ${ }^{1}$

- They display desirable dimensional characteristics and may be cast accurately ${ }^{[5,3]}$.

- More retentive ${ }^{[1]}$.

- Reduced displacement during lateral mandibular movements.

\section{Disadvantages of metal denture base}

- Increased cost, difficult to reline and rebase ${ }^{[5]}$.

- Indicated when polymer-based systems fail to provide acceptable physical properties.

- Time consuming requiring complex processing steps.

- Encroachment of interocclusal space.

\section{Case report}

A 76 years old male patient reported to the Department of prosthodontics and crown and bridge, Government Dental College, Thiruvananthapuram, with the chief complaint of repeated fracture of the upper denture from the middle and wants the denture that lasts long due to repeated fractures. Patient has a medical history of prostate cancer which was removed 12 years ago. Patient has past dental history of extractions of the grossly decayed and mobile teeth due to chronic generalized periodontitis.

Intraoral examination reveals complete edentulous of the maxillary arch with high frenum attachment and partial edentulous of the mandibular arch. In the mandibular arch only few remaining natural teeth were present. After evaluation of the intraoral examination, the treatment planning was explained to the patient. The patient was agreed for the best possible treatment.

Due to frequent fracture of the maxillary denture, planned to give stainless steel mesh incorporated maxillary denture and conventional removable partial denture for the mandibular arch.

\section{Materials and Methods}

- Impression compound

- Alginate impression materials

- Green stick compound

- Polyvinyl siloxane material

- Autopolymerizing acrylic resin

- Hot water

- Rubber bowl

- Spatula

- Modeling wax

- Teeth set

\section{Procedure}

Initially patient was seated comfortably in the dental chair. Then impressions were made with impression compound for the maxillary arch and with irreversible hydrocolloid impression materials for the mandibular arch. Impressions were poured in plaster of paris for the maxillary arch impressions and dental stone for the mandibular arch impressions.

Special tray was fabricated on the maxillary cast with the selfcure acrylic resins then it was placed in the patient's mouth and checked for $2 \mathrm{~mm}$ clearance between the tray and the tissue so that it should be recorded properly. Border moulding was recorded with green stick compound and secondary impressions were made with polyvinyl siloxane material. Impression was poured with dental stone. Denture base was fabricated with the help of shellac base plate in the maxillary cast and with the help of autopolymerizing acrylic resin in the mandibular cast. Then occlusal rims were adapted over both the denture base according to proper height and width.

Maxillary occlusal rims placed in the patients mouth and checked for the plane of occlusion in relation to inter pupillary line for the anterior plane and ala-tragus line for the posterior plane with fox plane and checked for lip fullness and buccal corridor after correction of maxillary occlusal rims, then mandibular occlusal rims placed in the patients mouth and checked for the plane of occlusion. After necessary adjustments, jaw relation was recorded.

Teeth were selected on basis of SPA concept. Teeth arrangement was done on the mean value articulator. After trial denture, necessary correction was done on the patient's mouth.

De-articulation of the cast from the articular done.

Clasps were made for mandibular removable partial denture base, then wax up and carving done. While waxing up of maxillary denture base, one layer of modeling wax that is thickness of modeling wax is adapted so that after dewaxing, there will be sufficient space for the stainless steel metal mesh to be adapted. Investing was done of both maxillary and mandibular cast. Dewaxing was done. Then a stainless steel metal mesh was adapted over the maxillary cast and fixed the mesh to the cast using a biocompatible glue so that mesh will not move while packing with heat cure acrylic resin. Packing was done and acrylization completed. Once the finishing and polishing was done, the prosthesis was inserted into the patient's mouth.

Instructions were given to the patient about proper removal and insertion of the denture and also advised to maintain a good oral hygiene by proper cleaning of the prosthesis after every meal.

Follow-up of the patient was done after 24 hours and no redness, pain or irritation in the soft tissue noticed.

\section{Discussion}

The most commonly used materials for the fabrication of removable complete or partial denture is PMMA based acrylic resin which has certain advantages that is low cost and easy to manipulate. The disadvantage is that it has poor strength which includes low impact strength and low fatigue resistance. Denture fractures result from two different types of forces namely flexural fatigue and impact. When opposing natural dentitions are present, flexural fatigue occurs due to excessive masticatory forces leads to repeated loads in the denture base due to which there is development of micro cracks in the denture base which results in fracture of the prosthesis. Midline fracture of the denture is a typical example of flexural fatigue. Impact failures happen outside the mouth due to accident while cleaning or coughing or even road traffic accident. As there has been reported with midline fractures of the denture are either due to the high palatal vault, bony prominences, prominent incisive papilla or prominent rugae. The best way to counteract these problems by using a metal mesh reinforcement in the prosthesis inspite of its disadvantages of increased cost, complex fabrication procedure and inability to reline and rebase.

But anyhow, patient was happy with the prosthesis as it is more rigid and retentive due to its reinforced stainless steel 
mesh denture base with good patient compliance.

\section{Conclusion}

Stainless steel metal reinforced maxillary denture is the best treatment options for the patient getting frequent fracture of the conventional denture especially in the midline. Stainless steel metal mesh denture has an added advantage that it increases the strength and durability of the prosthesis and at the same time provides satisfaction for the patient functionally. Due to repeated fractures, a patient faces problems with function and esthetics. So to overcome these issues, a stainless steel metal mesh reinforced maxillary complete denture was a treatment options to reduce the subsequent visits to the dentist and moreover very effective functionally and esthetically. Hence overall such prosthesis enhances the patient's confidence and body language.

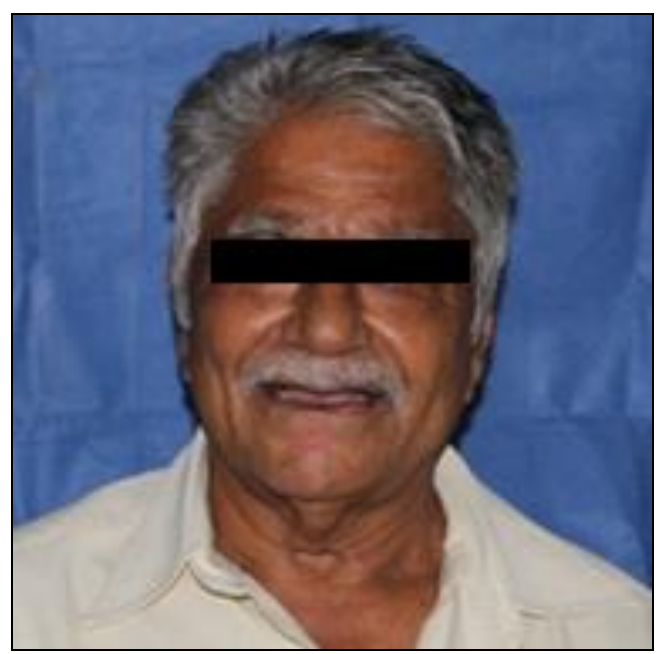

Fig 1: Profile Pic

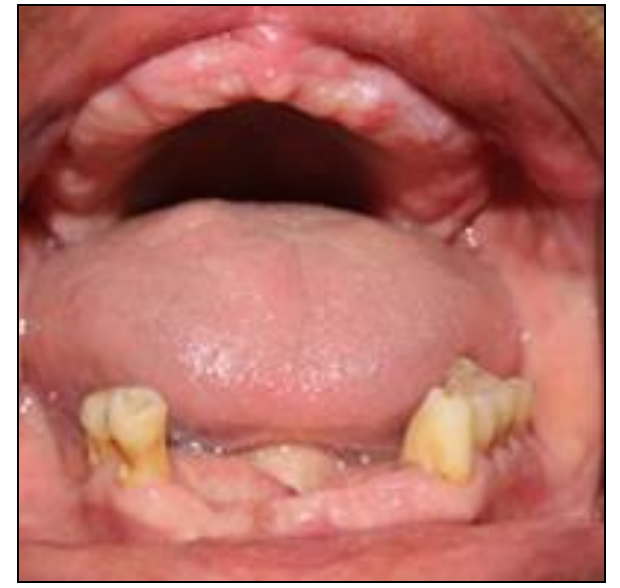

Fig 2: Intraoral views of the maxillary and mandibular arch

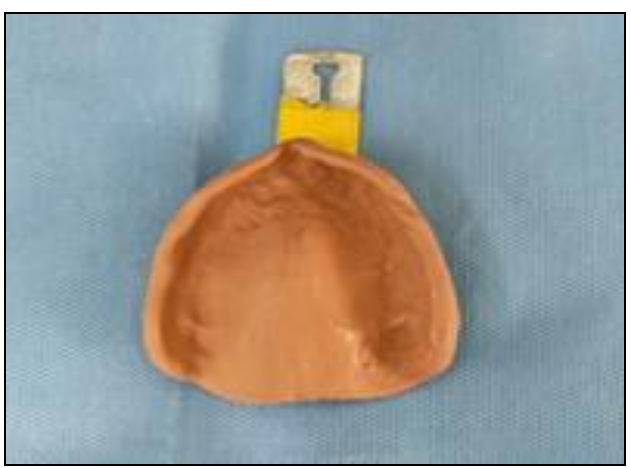

Fig 3: Primary Impression

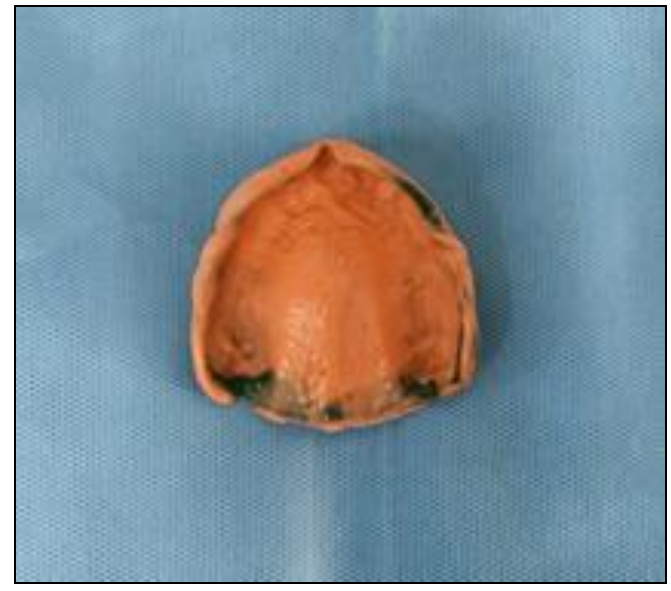

Fig 4: Secondary Impression

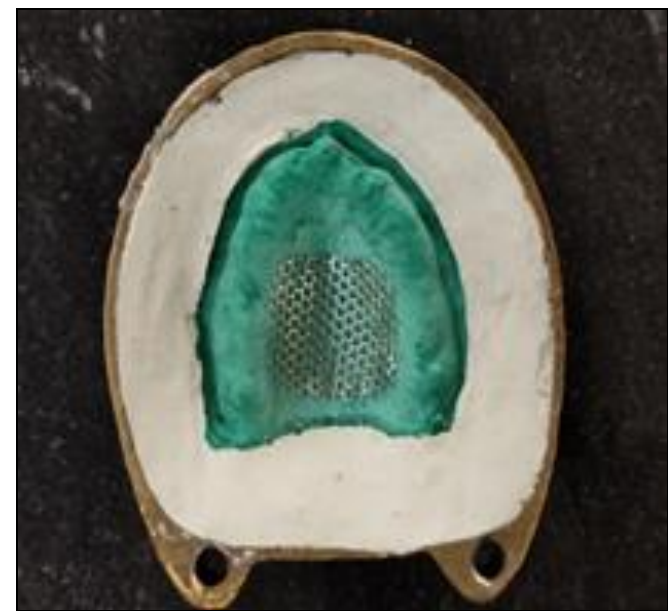

Fig 5: Metal mash adapted over the maxillary cast

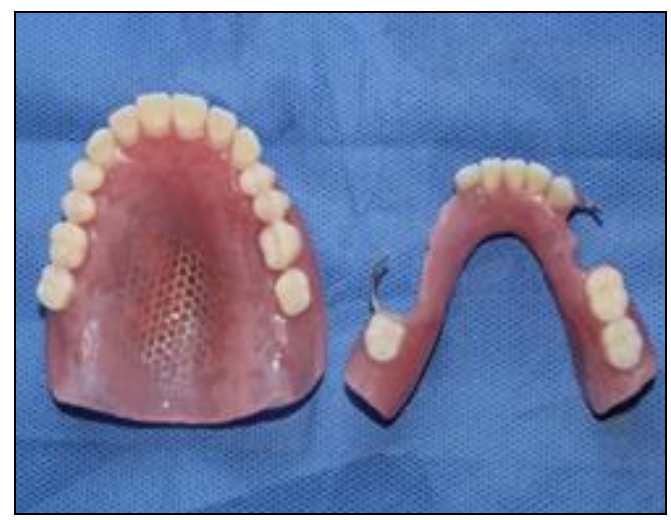

Fig 6: Finished Prosthesis

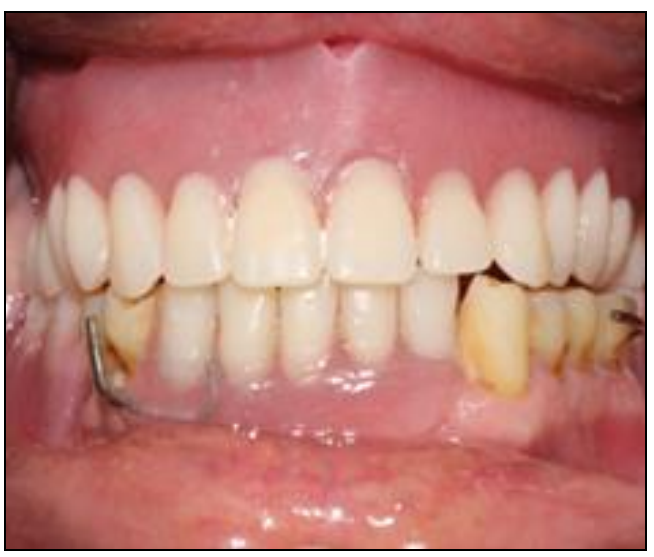

Fig 7: Maxillary CD and mandibular RPD in situ 


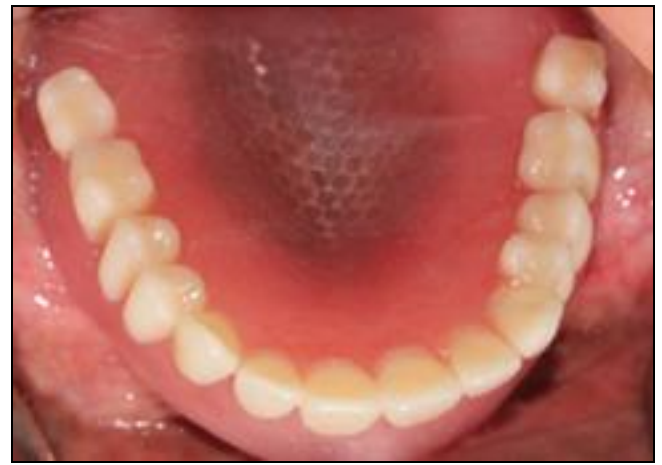

Fig 8: Prosthesis with mesh in situ

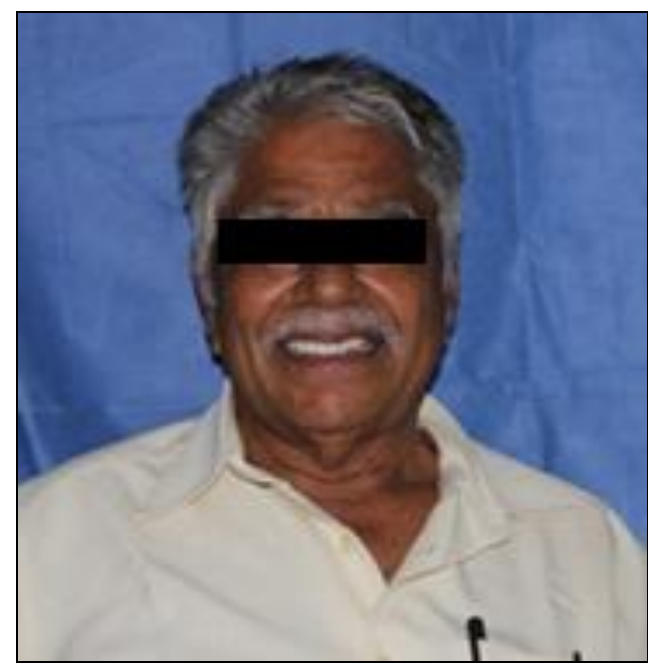

Fig 8: Final Prosthesis delivered

\section{References}

1. Rathod N, PawarS, Naitam D, Pasam N. Metal denture base an approach to overcome the failure of acrylic denture bases. IJRID 2015;5(2):69-72.

2. Prasad A, Prasad K and Hegde C. Management of completely edentulous maxillary arch opposing natural dentition- A case report. NUJHS 2012;2(3):39-42.

3. Jameson WS. Fabrication and use of a metal reinforcing frame in a fracture prone mandibular complete denture. 2000; 83(4):476-480.Jagger DC, Harrison A and Jandt KD. Review the reinforcement of dentures 1999;26:185194.

4. Darbar UR, Hugget R, Harrison A. Denture fracture: A survey. Br Dent J 1994;176:342-345.

5. Jagger DC, Harrison A and Jandt KD. Review the reinforcement of dentures 1999;26:185-194. 